\title{
Efficient Molecular Biological Manipulations with Improved Strategies Based on Novel Escherichia coli Vectors
}

Yifeng Zhang a,b $\S$, Herui Gao b ${ }^{b}$, Xianghui Qi ${ }^{c} \S$, Song Gao b, Shumin Xu a,b, Hongling Wang a,b, Yingfeng An a,b\#

a College of Food Science, Shenyang Agricultural University, Shenyang, China

${ }^{b}$ College of Biosciences and Biotechnology, Shenyang Agricultural University, Shenyang, China

c School of Food and Biological Engineering, Jiangsu University, Zhenjiang, China

$\S$ These authors contributed equally to this work

\# Corresponding author: Yingfeng An

Email: anyingfeng666@163.com

Address: College of Bioscience and Biotechnology, Shenyang Agricultural University. No.120 Dongling Road, Shenyang 110161, P. R. China.

Tel: +86-24-88487163. Fax: +86-24-88487163 


\section{Supplementary Table S1: Plasmids constructed in this study}

\begin{tabular}{|c|c|}
\hline Plasmids & Features \\
\hline pBV220-pfLamA & $\begin{array}{l}\text { Plasmid with pfLamA gene inserted into pBV220 vector for } \\
\text { temperature-induced protein expression }\end{array}$ \\
\hline pETM11-pfLamA & $\begin{array}{l}\text { Plasmid with pfLamA gene inserted into pETM11 vector for } \\
\text { IPTG-induced protein expression }\end{array}$ \\
\hline pANY4 & $\begin{array}{l}\text { Plasmid containing } \mathrm{T} 7 \text { promoter and } \mathrm{pR} 18 / \mathrm{pL} 18 \text { in the } \\
\text { complementary DNA strands for IPTG-induced or temperature- } \\
\text { induced protein expression }\end{array}$ \\
\hline $\begin{array}{l}\text { pANY4-pR/pL- } \\
\text { pfLamA }\end{array}$ & $\begin{array}{l}\text { Plasmid with pfLamA gene inserted into pANY4 vector in the } \\
\text { reverse direction for temperature-induced protein expression }\end{array}$ \\
\hline pANY4-T7-pfLamA & $\begin{array}{l}\text { Plasmid with pfLamA gene inserted into pANY4 vector in the } \\
\text { forward direction for IPTG-induced protein expression }\end{array}$ \\
\hline pANY4-pR/pL-GFP & $\begin{array}{l}\text { Plasmid with GFP gene inserted into pANY4 vector in the reverse } \\
\text { direction for temperature-induced protein expression }\end{array}$ \\
\hline pANY4-T7-GFP & $\begin{array}{l}\text { Plasmid with GFP gene inserted into pANY4 vector in the forward } \\
\text { direction for IPTG-induced protein expression }\end{array}$ \\
\hline pANY4-pR-GFP & $\begin{array}{l}\text { pANY4-backbone derived plasmid containing } \mathrm{pR} \text { promoter and } \\
\text { reverse insertion of GFP gene for temperature-induced protein } \\
\text { expression }\end{array}$ \\
\hline pANY4-pL-GFP & $\begin{array}{l}\text { pANY4-backbone derived plasmid containing } \mathrm{pL} \text { promoter and } \\
\text { reverse insertion of GFP gene for temperature-induced protein } \\
\text { expression }\end{array}$ \\
\hline $\begin{array}{l}\text { pANY4-pR9/pL18- } \\
\text { GFP }\end{array}$ & $\begin{array}{l}\text { pANY4-backbone derived plasmid containing } \mathrm{pR} 9 / \mathrm{pL} 18 \text { mutated } \\
\text { promoter and reverse insertion of GFP gene for temperature- } \\
\text { induced protein expression }\end{array}$ \\
\hline $\begin{array}{l}\text { pANY4-pR18/pL18- } \\
\text { GFP }\end{array}$ & $\begin{array}{l}\text { pANY4-backbone derived plasmid containing pR18/pL18 } \\
\text { mutated promoter and reverse insertion of GFP gene for } \\
\text { temperature-induced protein expression }\end{array}$ \\
\hline $\begin{array}{l}\text { pANY4-pR9/pL15- } \\
\text { GFP }\end{array}$ & $\begin{array}{l}\text { pANY4-backbone derived plasmid containing pR18/pL18 } \\
\text { mutated promoter and reverse insertion of GFP gene for } \\
\text { temperature-induced protein expression }\end{array}$ \\
\hline $\begin{array}{l}\text { pANY4-pR18/pL15- } \\
\text { GFP }\end{array}$ & $\begin{array}{l}\text { pANY4-backbone derived plasmid containing pR18/pL18 } \\
\text { mutated promoter and reverse insertion of GFP gene for } \\
\text { temperature-induced protein expression }\end{array}$ \\
\hline $\begin{array}{l}\text { pANY4-pR18/pL18- } \\
\text { pfLamA }\end{array}$ & $\begin{array}{l}\text { pANY4-backbone derived plasmid containing pR18/pL18 } \\
\text { mutated promoter and reverse insertion of GFP gene for } \\
\text { temperature-induced protein expression }\end{array}$ \\
\hline pANY4-pR-pfLamA & $\begin{array}{l}\text { pANY4-backbone derived plasmid containing } \mathrm{pR} \text { promoter and } \\
\text { reverse insertion of pfLamA gene for temperature-induced } \\
\text { protein expression }\end{array}$ \\
\hline pANY4-pR18- & pANY4-backbone derived plasmid containing pR18 mutated \\
\hline
\end{tabular}




\begin{tabular}{|l|l|}
\hline pfLamA & $\begin{array}{l}\text { promoter and reverse insertion of pfLamA gene for temperature- } \\
\text { induced protein expression }\end{array}$ \\
\hline pANY4-pL-pfLamA & $\begin{array}{l}\text { pANY4-backbone derived plasmid containing pL promoter and } \\
\text { reverse insertion of pfLamA gene for temperature-induced } \\
\text { protein expression }\end{array}$ \\
\hline $\begin{array}{l}\text { pANY4-pL18- } \\
\text { pfLamA }\end{array}$ & $\begin{array}{l}\text { pANY4-backbone derived plasmid containing pL18 mutated } \\
\text { promoter and reverse insertion of pfLamA gene for temperature- } \\
\text { induced protein expression }\end{array}$ \\
\hline pANY4-pL18-ccdB & $\begin{array}{l}\text { pANY4-backbone derived plasmid containing pL18 mutated } \\
\text { promoter and insertion of ccdB cassette for background-free } \\
\text { molecular cloning and bidirectional protein expression }\end{array}$ \\
\hline $\begin{array}{l}\text { pANY4-pR18/pL18- } \\
\text { ccdB }\end{array}$ & $\begin{array}{l}\text { pANY4-backbone derived plasmid containing pR18/pL18 } \\
\text { mutated promoter and insertion of ccdB cassette for background- } \\
\text { free molecular cloning and bidirectional protein expression }\end{array}$ \\
\hline pANY8 & $\begin{array}{l}\text { Plasmid containing ccdB cassette and a multifunctional fusion } \\
\text { tag for background-free molecular cloning, protein expression } \\
\text { and His-ELP-intein-based protein purification }\end{array}$ \\
\hline pANY8-pfLamA & $\begin{array}{l}\text { Plasmid with pfLamA gene inserted into pANY8 vector for protein } \\
\text { expression and His-ELP-intein-based protein purification }\end{array}$ \\
\hline
\end{tabular}

\section{Sequence 1 (DNA sequence of pANY4):}

ctcgttgaccctgagcaggctgttgagccaggtgatttctgcatagccagacttgggggtgatgagtttaccttcaagaaact gatcagggatagcggtcaggtgttttacaaccactaaacccacagtacccaatgatcccatgcaatgagagttgttccgttgt ggggaaagttatcgctagtcagtggcctgaagagacgtttggctgattggctgctgccaccgctgagcaataactagcataa ccccttggggectctaaacgggtcttgaggggtttttgctgaaaggaggaactatatccggatatccctgactgcgttagcaat ttaactgtgataaactaccgcattaaagcttatcgatgataagctgtcaaacatgagaattcttagaaaaactcatcgagcatc aaatgaaactgcaatttattcatatcaggattatcaataccatattttgaaaaagccgtttctgtaatgaaggagaaaactcac cgaggcagttccataggatggcaagatcctggtatcggtctgcgattccgactcgtccaacatcaatacaacctattaatttcc cctcgtcaaaaataaggttatcaagtgagaaatcaccatgagtgacgactgaatccggtgagaatggcaaaagtttgtgcat ttcttccagacttgttcaacaggccagccattacgctcgtcatcaaaatcactcgcatcaaccaaaccgttattcattcgtgatt gcgcctgagcgagacgaaatacgcgatcgctgttaaaaggacaattacaaacaggaatcgaatgcaaccggcgcagg aacactgccagcgcatcaacaatatttcacctgaatcaggatattcttctaatacctggaatgctgtttcccggggatcgcag tggtgagtaaccatgcgtcatcaggagtacggataaaatgcttgatggtcggaacaggcataaattccgtcagccagtttagt ctgaccatctcatctgtaacatcattggcaacgctacctttgccatgtttcagaaacaactctggcgcatcgggcttcccataca atcgatagattgtcgcacctgattgcccgacattatcgcgagcccatttatacccatataaatcagcatccatgttggaatttaat cgcggcctcgagcaagacgtttcccgttgaatatggctcataacaccccttgtattactgtttatgtaagcagacagtttattgtt catgaccaaaatcccttaacgtgagttttcgttccactgagcgtcagaccccgtagaaaagatcaaaggatcttcttgagatc cttttttctgcgcgtaatctgctgcttgcaaacaaaaaaaccaccgctaccagcggtggtttgtttgccggatcaagagctacc aactcttttccgaaggtaactggcttcagcagagcgcagataccaaatactgtccttctagtgtagccgtagttaggccacca cttcaagaactctgtagcaccgcctacatacctcgctctgctaatcctgttaccagtggctgctgccagtggcgataagtcgtgt cttaccgggttggactcaagacgatagttaccggataaggcgcagcggtcgggctgaacggggggttcgtgcacacagcc cagcttggagcgaacgacctacaccgaactgagatacctacagcgtgagctatgagaaagcgccacgcttcccgaagg gagaaaggcggacaggtatccggtaagcggcagggtcggaacaggagagcgcacgagggagcttccagggggaaa 
cgcctggtatctttatagtcctgtcgggtttcgccacctctgacttgagcgtcgattttgtgatgctcgtcaggggggcggagcct atggaaaaacgccagcaacgcggccttttacggttcctggcctttgctggcctttggtaccagatctcgatcccgcgaaatta atacgactcactatagggagaccacaacggtttccctctagaaataattttgtttaactttaagaaggagatatacatatgggc caccatcatcaccaccatgattatgatattccaactaccgagaatttgtatttcaggggggatccactagtaggcctgtcgac ggtcagtccggcttactaaaagccagataacagtatgcgtatttgcgcgctgattttgcggtataagaatatatactgatatgta tacccgaagtatgtcaaaaagaggtatgctatgaagcagcgtattacagtgacagttgacagcgacagctatcagttgctca aggcatatatgatgtcaatatctccggtctggtaagcacaaccatgcagaatgaagcccgtcgtctgcgtgccgaacgctgg aaagcggaaaatcaggaagggatggctgaggtcgcccggtttattgaaatgaacggctctttgctgacgagaacagggg ctggtgaaatgcagtttaaggtttacacctataaaagagagagccgttatcgtctgttgtggatgtacagagtgatattattgac acgcccgggcgacggatggtgatcccctggccagtgcacgtctgctgtcagataaagtctcccgtgaactttacccggtgg tgcatatcggggatgaaagctggcgcatgatgaccaccgatatggccagtgtgccggtctccgttatcggggaagaagtgg ctgatctcagccaccgcgaaaatgacatcaaaaacgccattaacctgatgttctggggaatataaatgtcaggctcccttata cacagccagtcgactgaccgtcaggcctccatgggagctcctgcaggtgatggtgatggtgatgcatatgtatatctccttaat ttttaaccaatgcttcgtttcgtatcacacaccccaaagccttctgctttgaatgctgcccttcttcagggcttaattttaagagcgt caccttcatggtggtcagtgcgtcctgctgatgtgctcagtatcaccgccagtggtatttatgtcaacaccgccagagataattt atcaccgcagatggttatctgtatgtttttatatgaatttatttttgcaggggggcattgtttggtaggtgagagatctttagctgtctt ggtttgcccaaagcgcattgcataatctttcagggttatgcgttgttccatacaacctccttagtacatgcaaccattatcaccgc cagaggtaaaatagtcaacacgcacggtgttagatatttatcccttgcggtgatagatttaacgtatgagcacaaaaaagaa accattaacacaagagcagcttgaggacgcacgtcgccttaaagcaatttatgaaaaaaagaaaaatgaacttggcttatc ccaggaatctgtcgcagacaagatggggatggggcagtcaggcgttggtgctttatttaatggcatcaatgcattaaatgctta taacgccgcattgcttacaaaaattctcaaagttagcgttgaagaatttagcccttcaatcgccagagaaatctacgagatgt atgaagcggttagtatgcagccgtcacttagaagtgagtatgagtaccctgtttttctcatgttcaggcagggatgttctcacct aagcttagaacctttaccaaaggtgatgcggagagatgggtaagcacaaccaaaaaagccagtga

Note: The highlighted sequences are $T 7$ promoter, DNA insert of ccdB cassette + multiple cloning sites, and $\mathrm{pR} / \mathrm{pL}$ promoter, respectively.

\section{Supplementary Table S2: pANY4-backbone derived plasmids}

\begin{tabular}{|l|l|l|}
\hline Plasmids & $\begin{array}{l}\text { The replaced } \\
\text { regions }\end{array}$ & $\begin{array}{l}\text { The DNA sequences used to construct pANY4- } \\
\text { backbone derived plasmids through replacing one } \\
\text { or two regions of pANY4 (highlighted with the } \\
\text { same colors as the corresponding regions in } \\
\text { Sequence 1) }\end{array}$ \\
\hline $\begin{array}{l}\mathrm{pANY4-} \\
\mathrm{pR} / \mathrm{pL}- \\
\mathrm{pfLamA}\end{array}$ & $\begin{array}{l}\text { DNA insert of } \\
\text { pfLamA in the } \\
\text { reverse direction } \\
\text { + multiple cloning } \\
\text { sites }\end{array}$ & $\begin{array}{l}\text { ggatccactagtaggctgtcgacggtaggttaaccactaacgaatga } \\
\text { gtaaaccttacataatccaccaccatcttgctggaaatggagttgtag } \\
\text { catcggggtttcctggccaataaccacccactgcaagattaaggattat } \\
\text { atagaagggcttatcgaagacccactcatagccatagcctccacttgtt } \\
\text { ctttgtaacctcatgataaaaagttccatcaacgtaccactttatttatcc } \\
\text { ggataccaaactattccaaatacatggaagtcttctgtaaagtctggaac } \\
\text { accttcagggagtgtataggccctagtaattcctttacttcccgagtaacc } \\
\text { tgggccatgaacagttccgtgaattgtccgtggctcatggccaaggaac } \\
\text { tccattatgtctatttctccacaatttggccagcctacttcccttatgttgctcc }\end{array}$ \\
\hline
\end{tabular}




\begin{tabular}{|c|c|c|}
\hline & & $\begin{array}{l}\text { ccaacatccagaatgcaggccataaacctttaccttttggaagctttattc } \\
\text { tagcctcaacaactactggagggctaaattctaccttaccttcagtcttaa } \\
\text { gtcttgatgaagtgtagagaaacgttccttcgttaggatcagtaattatttct } \\
\text { tttctggcctcaatgacaagggtgccatttacaatataggtgttgtttccgt } \\
\text { gtagtattcaagctctccattcccccatcccgggattccataagctattcc } \\
\text { atttcccttctcaaatgtccagtattctttatttacttcggaaccttcaaactca } \\
\text { tcgtgccaaataagtctccactgttttccatctatttctatcacttcagggac } \\
\text { catgccaccgtcaggcctccatgggagctcctgcag }\end{array}$ \\
\hline $\begin{array}{l}\text { pANY4-T7- } \\
\text { pfLamA }\end{array}$ & $\begin{array}{l}\text { DNA insert of } \\
\text { pfLamA in the } \\
\text { forward direction } \\
+ \text { multiple cloning } \\
\text { sites }\end{array}$ & $\begin{array}{l}\text { ggatccactagtaggcctgtcgacggtggcatggtccctgaagtgatag } \\
\text { aaatagatggaaaacagtggagacttatttggcacgatgagtttgaag } \\
\text { gttccgaagtaaataaagaatactggacattgagaagggaaatgga } \\
\text { atagcttatggaatcccgggatgggggaatggagagcttgaatactac } \\
\text { acggaaaacaacacctatattgtaaatggcacccttgtcattgaggcca } \\
\text { gaaaagaaataattactgatcctaacgaaggaacgtttctctacacttca } \\
\text { tcaagacttaagactgaaggtaaggtagaatttagccctccagtagttgt } \\
\text { tgaggctagaataaagcttccaaaaggtaaaggtttatggcctgcattct } \\
\text { ggatgttggggagcaacataagggaagtaggctggccaaattgtgga } \\
\text { gaaatagacataatggagttccttggccatgagccacggacaattcac } \\
\text { ggaactgttcatggcccaggttactcgggaagtaaaggaattactagg } \\
\text { gcctatacactccctgaaggtgttccagactttacagaagacttccatgt } \\
\text { atttggaatagtttggtatccggataaaataaagtggtacgttgatggaa } \\
\text { cttttatcatgaggttacaaaagaacaagtggaggctatgggctatgag } \\
\text { tgggtcttcgataagcccttctatataatccttaatcttgcagtgggtggttat } \\
\text { tggccaggaaaccccgatgctacaactccatttccagcaaagatggtg } \\
\text { gtggattatgtaagggtttactcattcgttagtggttaacctaccgtcaggc } \\
\text { ctccatgggagctcctgcag }\end{array}$ \\
\hline $\begin{array}{l}\text { pANY4- } \\
\text { pR/pL- } \\
\text { GFP }\end{array}$ & $\begin{array}{l}\text { DNA insert of } \\
\text { GFP in the } \\
\text { reverse direction } \\
+ \text { multiple cloning } \\
\text { sites }\end{array}$ & $\begin{array}{l}\text { ggatccactagtaggcctgtcgacggtaggttattactccttgtacagctc } \\
\text { gtccatgccgagagtgatcccggcggcggtcacgaactccagcagga } \\
\text { ccatgtgatcgcgcttctcgttggggtctttgctcagggcggactgggtgc } \\
\text { tcaggtagtggttgtcgggcagcagcacggggccgtcgccgatgggg } \\
\text { gtgttctgctggtagtggtcggcgagctgcacgctgccgtcctcgatgttg } \\
\text { tggcggatcttgaagttcaccttgatgccgttcttctgcttgtcggccatgat } \\
\text { atagacgttgtggctgttgtagttgtactccagcttgtgccccaggatgttg } \\
\text { ccgtcctccttgaagtcgatgcccttcagctcgatgcggttcaccagggt } \\
\text { gtcgccctcgaacttcacctcggcgcgggtcttgtagttgccgtcgtcctt } \\
\text { gaagaagatggtgcgctcctggacgtagccttcgggcatggcggactt } \\
\text { gaagaagtcgtgctgcttcatgtggtcggggtagcggctgaagcactgc } \\
\text { acgccgtaggtcagggtggtcacgagggtgggccagggcacgggca } \\
\text { gcttgccggtggtgcagatgaacttcagggtcagcttgccgtaggtggc } \\
\text { atcgccctcgccctcgccggacacgctgaacttgtggccgtttacgtcgc } \\
\text { cgtccagctcgaccaggatgggcaccaccccggtgaacagctcctcg } \\
\text { cccttgctcaccatgccaccgtcaggcctccatgggagctcctgcag }\end{array}$ \\
\hline $\begin{array}{l}\text { pANY4-T7- } \\
\text { GFP }\end{array}$ & $\begin{array}{l}\text { DNA insert of } \\
\text { GFP in the }\end{array}$ & $\begin{array}{l}\text { ggatccactagtaggcctgtcgacggtaggttaatggtgagcaagggc } \\
\text { gaggagctgttcaccggggtggtgcccatcctggtcgagctggacggc }\end{array}$ \\
\hline
\end{tabular}




\begin{tabular}{|c|c|c|}
\hline & $\begin{array}{l}\text { forward direction } \\
+ \text { multiple cloning } \\
\text { sites }\end{array}$ & $\begin{array}{l}\text { gacgtaaacggccacaagttcagcgtgtccggcgagggcgagggcg } \\
\text { atgccacctacggcaagctgaccctgaagttcatctgcaccaccggca } \\
\text { agctgcccgtgccctggcccaccctcgtgaccaccctgacctacggcg } \\
\text { tgcagtgcttcagccgctaccccgaccacatgaagcagcacgacttctt } \\
\text { caagtccgccatgcccgaaggctacgtccaggagcgcaccatcttctt } \\
\text { caaggacgacggcaactacaagacccgcgccgaggtgaagttcga } \\
\text { gggcgacaccctggtgaaccgcatcgagctgaagggcatcgacttca } \\
\text { aggaggacggcaacatcctggggcacaagctggagtacaactacaa } \\
\text { cagccacaacgtctatatcatggccgacaagcagaagaacggcatc } \\
\text { aaggtgaacttcaagatccgccacaacatcgaggacggcagcgtgc } \\
\text { agctcgccgaccactaccagcagaacaccccatcggcgacggccc } \\
\text { cgtgctgctgcccgacaaccactacctgagcacccagtccgccctgag } \\
\text { caaagaccccaacgagaagcgcgatcacatggtcctgctggagttcg } \\
\text { tgaccgccgccgggatcactctcggcatggacgagctgtacaaggag } \\
\text { taagccaccgtcaggcctccatgggagctcctgcag }\end{array}$ \\
\hline \multirow[t]{2}{*}{$\begin{array}{l}\text { pANY4- } \\
\text { pR-GFP }\end{array}$} & $\begin{array}{l}\text { DNA insert of } \\
\text { GFP in the } \\
\text { reverse direction } \\
+ \text { multiple cloning } \\
\text { sites }\end{array}$ & The same as that of pANY4-pR/pL-GFP \\
\hline & pR promoter & $\begin{array}{l}\text { gcaggggggcattgtttggtaggtgagagatctttagctgtcttggtttgcc } \\
\text { caaagcgcattgcataatctttcagggttatgcgttgttccatacaacctc } \\
\text { cttagtacatgcaaccattatcaccgccagaggtaaaatagtcaacac } \\
\text { g }\end{array}$ \\
\hline \multirow[t]{2}{*}{$\begin{array}{l}\text { pANY4-pL- } \\
\text { GFP }\end{array}$} & $\begin{array}{l}\text { DNA insert of } \\
\text { GFP in the } \\
\text { reverse direction } \\
+ \text { multiple cloning } \\
\text { sites }\end{array}$ & The same as that of pANY4-pR/pL-GFP \\
\hline & pL promoter & $\begin{array}{l}\text { gtcaccttcatggtggtcagtgcgtcctgctgatgtgctcagtatcaccgc } \\
\text { cagtggtatttatgtcaacaccgccagagataatttatcaccgcagatgg } \\
\text { ttatctgtatgtttttatatgaatttatttttgcaggggggcattgtttggtagg } \\
\text { tgaga }\end{array}$ \\
\hline \multirow[t]{2}{*}{$\begin{array}{l}\text { pANY4- } \\
\text { pR9/pL18- } \\
\text { GFP }\end{array}$} & $\begin{array}{l}\text { DNA insert of } \\
\text { GFP in the } \\
\text { reverse direction } \\
+ \text { multiple cloning } \\
\text { sites }\end{array}$ & The same as that of pANY4-pR/pL-GFP \\
\hline & $\begin{array}{l}\text { Mutated } \\
\text { pR9/pL18 } \\
\text { promoter }\end{array}$ & $\begin{array}{l}\text { gtcaccttcatggtggtcagtgcgtcctgctgatgtgctcagtatcaccgct } \\
\text { agtggtatttctgtcaacaccgccagagataatttatcaccgcagatggtt } \\
\text { atctgtatgtttttatatgaatttatttttgcaggggggcattgtttggtaggt } \\
\text { gagagatctttagctgtcttggtttgcccaaagcgcattgcataatcttca } \\
\text { gggttatgcgttgttccatacaacctccttagtacatgcaaccattatcacc }\end{array}$ \\
\hline
\end{tabular}




\begin{tabular}{|c|c|c|}
\hline & & gccagaggtataacagtcaacacg \\
\hline \multirow[t]{2}{*}{$\begin{array}{l}\text { pANY4- } \\
\text { pR18/pL18 } \\
\text {-GFP }\end{array}$} & $\begin{array}{l}\text { DNA insert of } \\
\text { GFP in the } \\
\text { reverse direction } \\
+ \text { multiple cloning } \\
\text { sites }\end{array}$ & The same as that of pANY4-pR/pL-GFP \\
\hline & $\begin{array}{l}\text { Mutated } \\
\text { pR18/pL18 } \\
\text { promoter }\end{array}$ & $\begin{array}{l}\text { gtcaccttcatggtggtcagtgcgtcctgctgatgtgctcagtatcaccgct } \\
\text { agtggtatttctgtcaacaccgccagagataatttatcaccgcagatggtt } \\
\text { atctgtatgtttttatatgaatttattttttgcaggggggcattgtttggtaggt } \\
\text { gagagatctttagctgtcttggtttgcccaaagcgcattgcataatctttca } \\
\text { gggttatgcgttgttccatacaacctccttagtacatgcaaccattatcacc } \\
\text { gcaagaggtacaacagtcaacacg }\end{array}$ \\
\hline \multirow[t]{2}{*}{$\begin{array}{l}\text { pANY4- } \\
\text { pR9/pL15- } \\
\text { GFP }\end{array}$} & $\begin{array}{l}\text { DNA insert of } \\
\text { GFP in the } \\
\text { reverse direction } \\
+ \text { multiple cloning } \\
\text { sites }\end{array}$ & The same as that of pANY4-pR/pL-GFP \\
\hline & $\begin{array}{l}\text { Mutated } \\
\text { pR9/pL15 } \\
\text { promoter }\end{array}$ & $\begin{array}{l}\text { gtcaccttcatggtggtcagtgcgtcctgctgatgtgctcagtatcaccgc } \\
\text { cagtggtatttctgtcaacaccgccagagataatttatcaccgcagatgg } \\
\text { ttatctgtatgtttttatatgaatttatttttgcaggggggcattgtttggtagg } \\
\text { tgagagatctttagctgtcttggtttgcccaaagcgcattgcataatctttca } \\
\text { gggttatgcgttgttccatacaacctccttagtacatgcaaccattatcacc } \\
\text { gccagaggtataacagtcaacacg }\end{array}$ \\
\hline \multirow[t]{2}{*}{$\begin{array}{l}\text { pANY4- } \\
\text { pR18/pL15 } \\
\text {-GFP }\end{array}$} & $\begin{array}{l}\text { DNA insert of } \\
\text { GFP in the } \\
\text { reverse direction } \\
+ \text { multiple cloning } \\
\text { sites }\end{array}$ & The same as that of pANY4-pR/pL-GFP \\
\hline & $\begin{array}{l}\text { Mutated } \\
\text { pR18/pL15 } \\
\text { promoter }\end{array}$ & $\begin{array}{l}\text { gtcaccttcatggtggtcagtgcgtcctgctgatgtgctcagtatcaccgc } \\
\text { cagtggtatttctgtcaacaccgccagagataatttatcaccgcagatgg } \\
\text { ttatctgtatgtttttatatgaatttattttttgcaggggggcattgtttggtagg } \\
\text { tgagagatctttagctgtcttggtttgcccaaagcgcattgcataatctttca } \\
\text { gggttatgcgttgttccatacaacctccttagtacatgcaaccattatcacc } \\
\text { gcaagaggtacaacagtcaacacg }\end{array}$ \\
\hline \multirow[t]{2}{*}{$\begin{array}{l}\text { pANY4- } \\
\text { pR18/pL18 } \\
\text {-pfLamA }\end{array}$} & $\begin{array}{l}\text { DNA insert of } \\
\text { pfLamA in the } \\
\text { reverse direction } \\
+ \text { multiple cloning } \\
\text { sites }\end{array}$ & The same as that of pANY4-pR/pL-pfLamA \\
\hline & $\begin{array}{l}\text { Mutated } \\
\text { pR18/pL18 } \\
\text { promoter }\end{array}$ & The same as that of pANY4-pR18/pL18-GFP \\
\hline pANY4- & DNA insert of & The same as that of pANY4-pR/pL-pfLamA \\
\hline
\end{tabular}




\begin{tabular}{|c|c|c|}
\hline \multirow[t]{2}{*}{$\begin{array}{l}\text { pR- } \\
\text { pfLamA }\end{array}$} & $\begin{array}{l}\text { pflamA in the } \\
\text { reverse direction } \\
+ \text { multiple cloning } \\
\text { sites }\end{array}$ & \\
\hline & pR promoter & The same as that of pANY4-pR-GFP \\
\hline \multirow[t]{2}{*}{$\begin{array}{l}\text { pANY4- } \\
\text { pR18- } \\
\text { pfLamA }\end{array}$} & $\begin{array}{l}\text { DNA insert of } \\
\text { pfLamA in the } \\
\text { reverse direction } \\
+ \text { multiple cloning } \\
\text { sites }\end{array}$ & The same as that of pANY4-pR/pL-pfLamA \\
\hline & $\begin{array}{l}\text { Mutated } \quad \text { pR18 } \\
\text { promoter }\end{array}$ & $\begin{array}{l}\text { gcaggggggcattgtttggtaggtgagagatctttagctgtcttggtttgcc } \\
\text { caaagcgcattgcataatctttcagggttatgcgttgttccatacaacctc } \\
\text { cttagtacatgcaaccattatcaccgcaagaggtacaacagtcaacac } \\
\text { g }\end{array}$ \\
\hline \multirow[t]{2}{*}{$\begin{array}{l}\text { pANY4-pL- } \\
\text { pfLamA }\end{array}$} & $\begin{array}{l}\text { DNA insert of } \\
\text { pfLamA in the } \\
\text { reverse direction } \\
+ \text { multiple cloning } \\
\text { sites }\end{array}$ & The same as that of pANY4-pR/pL-pfLamA \\
\hline & pL promoter & The same as that of pANY4-pL-GFP \\
\hline \multirow[t]{2}{*}{$\begin{array}{l}\text { pANY4- } \\
\text { pL18- } \\
\text { pfLamA }\end{array}$} & $\begin{array}{l}\text { DNA insert of } \\
\text { pfLamA in the } \\
\text { reverse direction } \\
+ \text { multiple cloning } \\
\text { sites }\end{array}$ & The same as that of pANY4-pR/pL-pfLamA \\
\hline & $\begin{array}{l}\text { Mutated } \quad \text { pL18 } \\
\text { promoter }\end{array}$ & $\begin{array}{l}\text { gtcaccttcatggtggtcagtgcgtcctgctgatgtgctcagtatcaccgct } \\
\text { agtggtatttctgtcaacaccgccagagataatttatcaccgcagatggtt } \\
\text { atctgtatgtttttatatgaatttattttttgcaggggggcattgtttggtaggt } \\
\text { gaga }\end{array}$ \\
\hline $\begin{array}{l}\text { pANY4- } \\
\text { pL18-ccdB }\end{array}$ & $\begin{array}{l}\text { Mutated pL18 } \\
\text { promoter }\end{array}$ & The same as that of pANY4-pL18-pfLamA \\
\hline $\begin{array}{l}\text { pANY4- } \\
\text { pR18/pL18 } \\
\text {-ccdB }\end{array}$ & $\begin{array}{l}\text { Mutated } \\
\text { pR18/pL18 } \\
\text { promoter }\end{array}$ & The same as that of pANY4-pR18/pL18-GFP \\
\hline
\end{tabular}

\section{Sequence 2 (DNA sequence of pANY8):}

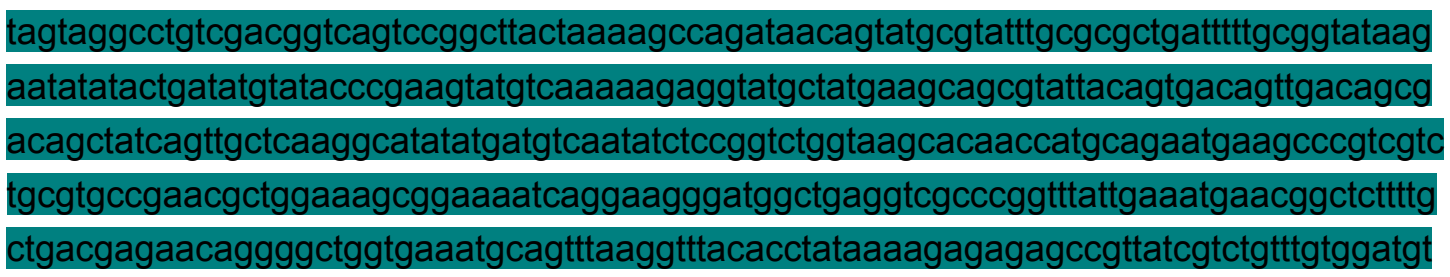



ctgagcaataactagcataaccccttggggcctctaaacgggtcttgaggggtttttgctgaaaggaggaactatatccgga tatccctgactgcgttagcaatttaactgtgataaactaccgcattaaagcttatcgatgataagctgtcaaacatgagaattctt agaaaaactcatcgagcatcaaatgaaactgcaatttattcatatcaggattatcaataccatattttgaaaaagccgtttctgt aatgaaggagaaaactcaccgaggcagttccataggatggcaagatcctggtatcggtctgcgattccgactcgtccaaca tcaatacaacctattaatttcccctcgtcaaaaataaggttatcaagtgagaaatcaccatgagtgacgactgaatccggtga gaatggcaaaagtttgtgcatttcttccagacttgttcaacaggccagccattacgctcgtcatcaaaatcactcgcatcaacc aaaccgttattcattcgtgattgcgcctgagcgagacgaaatacgcgatcgctgttaaaaggacaattacaaacaggaatc gaatgcaaccggcgcaggaacactgccagcgcatcaacaatatttcacctgaatcaggatattcttctaatacctggaatg ctgtttcccggggatcgcagtggtgagtaaccatgcgtcatcaggagtacggataaaatgcttgatggtcggaacaggcat aaattccgtcagccagtttagtctgaccatctcatctgtaacatcattggcaacgctaccttgccatgttcagaaacaactctg gcgcatcgggcttcccatacaatcgatagattgtcgcacctgattgcccgacattatcgcgagcccatttatacccatataaat cagcatccatgttggaatttaatcgcggcctcgagcaagacgtttcccgttgaatatggctcataacaccccttgtattactgttt atgtaagcagacagttttattgttcatgaccaaaatccttaacgtgagtttcgttccactgagcgtcagaccccgtagaaaag atcaaaggatcttcttgagatccttttttctgcgcgtaatctgctgcttgcaaacaaaaaaaccaccgctaccagcggtggtttg tttgccggatcaagagctaccaactcttttccgaaggtaactggcttcagcagagcgcagataccaaatactgtccttctagt gtagccgtagttaggccaccacttcaagaactctgtagcaccgcctacatacctcgctctgctaatcctgttaccagtggctgc tgccagtggcgataagtcgtgtcttaccgggttggactcaagacgatagttaccggataaggcgcagcggtcgggctgaac ggggggttcgtgcacacagcccagcttggagcgaacgacctacaccgaactgagatacctacagcgtgagctatgagaa agcgccacgcttcccgaagggagaaaggcggacaggtatccggtaagcggcagggtcggaacaggagagcgcacga gggagcttccagggggaaacgcctggtatctttatagtcctgtcgggtttcgccacctctgacttgagcgtcgattttgtgatgct cgtcaggggggcggagcctatggaaaaacgccagcaacgcggccttttacggttcctggccttttgctggcctttggtacca gatctcgatcccgcgaaattaatacgactcactatagggagaccacaacggtttccctctagaaataattttgtttaactttaag aaggagatatacatatgggccaccatcatcaccaccatgattatgatattccaactaccgagaatttgtatttcagggggtcc cggggaaaggcgtgcctggtgtcggggttccaggtgtgggtgtaccaggggtaggtgtaccaggtgtgggcgttccaggcg ttggtgtaccaggagtaggagtcccaggtgtaggcgtaccgggtttggagtacctggaaagggcgtcccaggggttggtgt acccggcgttggtgttcctggggtaggtgttccaggcgtaggagtaccaggcgttggagttcctggtgttggggtgcctggcgt tggggttcctggttttggtgtcccaggaaagggagttcctggcgtaggcgttccaggagtcggcgtaccgggagttggagttc caggagtaggcgttcctggagtcggagtacctggggtcggcgtgcctggagtcggtgttccaggattcggtgttcctggtaaa ggcgtcccaggagtgggtgttcctggcgtcggagtgcctggagtaggggttccoggtgtaggcgtgccoggagtaggtgta cctggagttggtgtaccgggagtaggtgtccctggtttcggagtccctggcaagggggtacccggagttggcgtaccgggg gtcggggtcccaggcgtcggtgtaccgggcgttggagtccccggtgtcggtgtgcccggcgtcggcgtgcccggtgtaggt gtgcctggtttcggtgtgccaggcaagggagtaccaggagtcggggtaccoggtgttggcgtaccoggggtgggggttcct ggagtgggagttccgggggtgggagtgcctggggtgggtgtaccoggggttggggtgccaggattcggagtgccgggga aaggtgttccgggtgtgggagtccccggcgtaggggtacctggtgtaggggtaccaggcgtaggtgtacccggggtcgga gttccaggtgtaggtgtaccgggtgtcggggtccccggattcggtgtgagctcgaacaacaacaacaataacaataacaac aacgaattcgccctcgcagagggcactcggatcttcgatccggtcaccggtacaacgcatcgcatcgaggatgttgtcggtg ggcgcaagcctattcatgtcgtggctgctgccaaggacggaacgctgcgtgcgcggcccgtggtgtcctggttcgaccagg gaacgcgggatgtgatcgggttgcggatcgccggtggcgccatcctgtgggcgacacccgatcacaaggtgctgacaga gtacggctggcgtgccgccggggaactccgcaagggagacagggtggcgcaaccgcgacgcttcgatggattcggtga cagtgcgccgattccggcgcgcgtgcaggcgctcgcggatgcctggatgacaaattcctgcacgacatgctggcggaag 
aactccgctattccgtgatccgagaagtgctgccaacgcggcggggacggacgttcggcctcgaggtcgaggaactgcac accctcgtcgccgaaggggttgttgtacacaacggatccac

Note: The highlighted sequences are His-tag, ELP, intein and DNA insert of ccdB cassette + multiple cloning sites, respectively.

\section{Sequence 3 (DNA sequence of pANY8-pfLamA):}

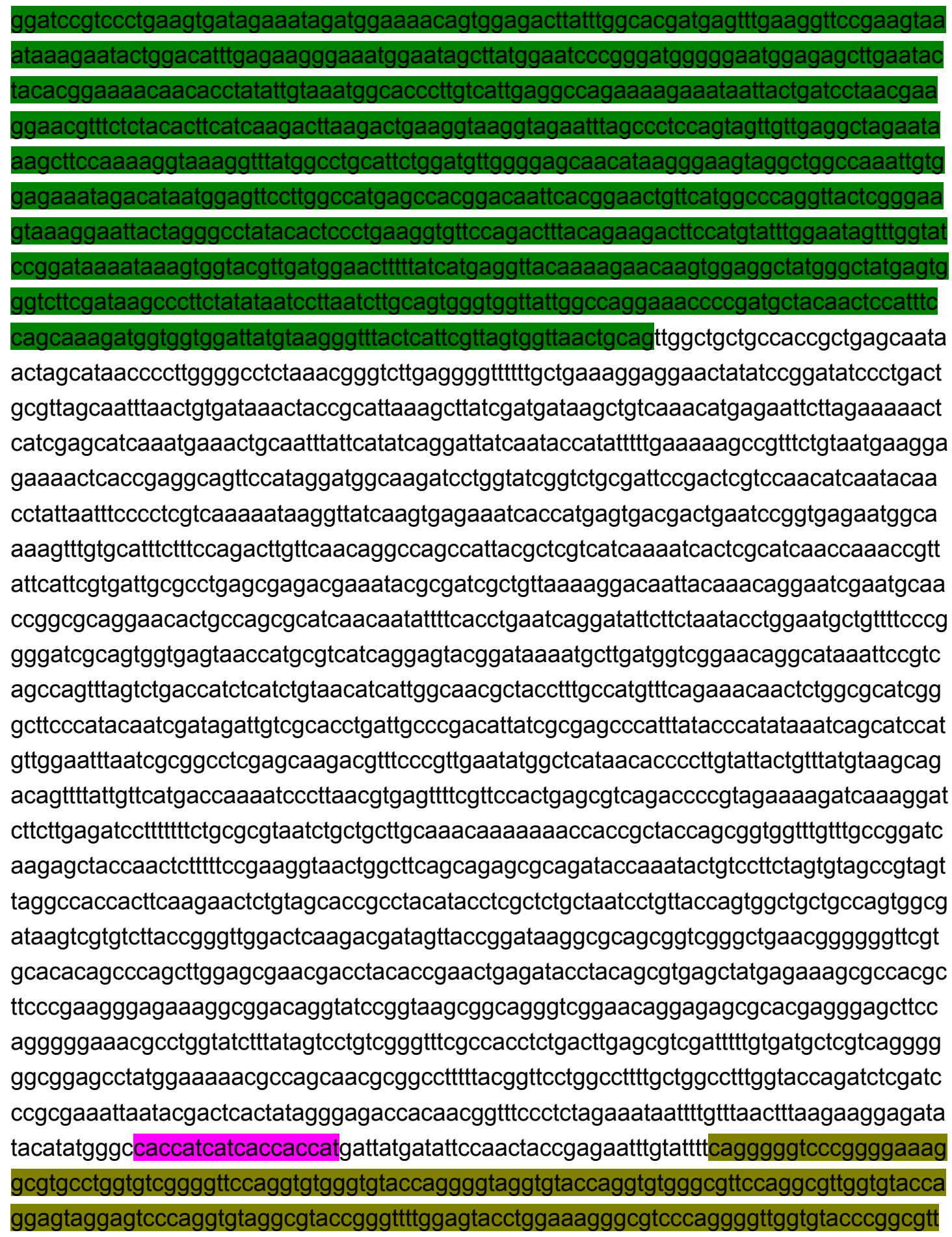


ggtgttcctggggtaggtgttccaggcgtaggagtaccaggcgttggagttcctggtgttggggtgcctggcgttggggttcctg

gtttggtgtcccaggaaagggagttcctggcgtaggcgttccaggagtcggcgtaccgggagttggagttccaggagtagg

cgttcctggagtcggagtacctggggtcggcgtgcctggagtcggtgttccaggattcggtgttcctggtaaaggcgtcccag

gagtgggtgttcctggcgtcggagtgcctggagtaggggttcccggtgtaggcgtgcccggagtaggtgtacctggagttggt

gtaccgggagtaggtgtccctggtttcggagtccctggcaagggggtacccggagttggcgtaccgggggtcggggtccca

ggcgtcggtgtaccgggcgttggagtccccggtgtcggtgtgcccggcgtcggcgtgcccggtgtaggtgtgcctggtttcgg

tgtgccaggcaagggagtaccaggagtcggggtaccgggtgttggcgtacccggggtgggggttcctggagtgggagttc

cgggggtgggagtgcctggggtgggtgtaccgggggttggggtgccaggattcggagtgccggggaaaggtgttccgggt

gtgggagtccccggcgtaggggtacctggtgtaggggtaccaggcgtaggtgtacccggggtcggagttccaggtgtaggt gtaccgggtgtcggggtccccggattcggtgtgagctcgaacaacaacaacaataacaataacaacaacgaattcgccct cgcagagggcactcggatcttcgatccggtcaccggtacaacgcatcgcatcgaggatgttgtcggtgggcgcaagcctat tcatgtcgtggctgctgccaaggacggaacgctgcgtgcgcggcccgtggtgtcctggttcgaccagggaacgcgggatgt gatcgggttgcggatcgccggtggcgccatcctgtgggcgacacccgatcacaaggtgctgacagagtacggctggcgtg ccgccggggaactccgcaagggagacagggtggcgcaaccgcgacgcttcgatggattcggtgacagtgcgccgattcc ggcgcgcgtgcaggcgctcgcggatgccctggatgacaaattcctgcacgacatgctggcggaagaactccgctattccgt gatccgagaagtgctgccaacgcggcgggcacggacgttcggcctcgaggtcgaggaactgcacaccctcgtcgccga aggggttgttgtacacaac

Note: The highlighted sequences are His-tag, ELP, intein and DNA insert of pfLamA + multiple cloning sites, respectively. 